\title{
Endoscopic esophagoplasty for megaesophagus with sump stasis in end-stage achalasia
}

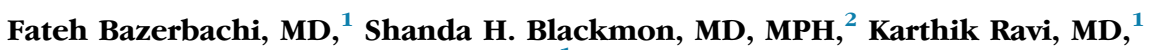
Louis M. Wong Kee Song, MD, FASGE ${ }^{1}$

End-stage disease develops in up to $5 \%$ of all achalasia patients despite primary endoscopic and surgical interventions. This is characterized by esophageal dilation with a diameter exceeding $6 \mathrm{~cm}$ and by esophageal tortuosity leading to a sigmoid-shaped megaesophagus. This may lead to deleterious adverse events, such as pulmonary aspiration of regurgitated food. The lower esophageal sphincter may be lax and not hypertonic. Therefore, myotomy is unlikely to improve esophageal emptying because the culprit pathophysiology is sump formation with food stasis. Esophagectomy may be the only option to address the culprit pathophysiology. ${ }^{1}$ This highly invasive procedure is associated with significant mortality and morbidity, and the surgical risk may be prohibitive if comorbidities are present. We propose a novel approach of endoscopic esophagoplasty to plicate and reduce the esophageal sump, leading to a straighter esophagus and improved esophageal emptying.

A 79-year-old woman who had undergone open surgical esophagomyotomy for achalasia in the 1970s presented with dysphagia, regurgitation, and aspiration symptoms, requiring several hospitalizations because of aspiration pneumonia, dehydration, and for endoscopic clearance of retained food in the past year. Endoscopy showed a food-filled megaesophagus and a nonhypertonic but angulated gastroesophageal junction (GEJ) resulting from sump formation in the distal esophagus (Fig. 1). An esophagram showed marked dilation of the distal esophagus and preferential contrast material pooling and stasis in the sump (Fig. 2). A repeated myotomy was not deemed beneficial in the setting of a lax GEJ and the dependent sump. The patient was not a candidate for esophagectomy because of her comorbidities.

A novel approach of endoscopic esophagoplasty was proposed to plicate and reduce the esophageal sump and, in doing so, straighten the esophagus to improve esophageal emptying. This was undertaken to improve the patient's quality of life and obviate the need for recurrent hospitalizations, because the patient's symptoms were not deemed to be related to inappropriate lower-esophageal sphincter dynamics. ${ }^{2}$

The lateral margins of the esophageal sump were marked with coagulation dots to serve as visual markers. Next, an endoscopic suturing device was used to place full-thickness running sutures in a triangular fashion to plicate the sump and straighten the esophagus. After esophagoplasty, sump reduction and improved esophageal emptying across the GEJ were noted by esophagography

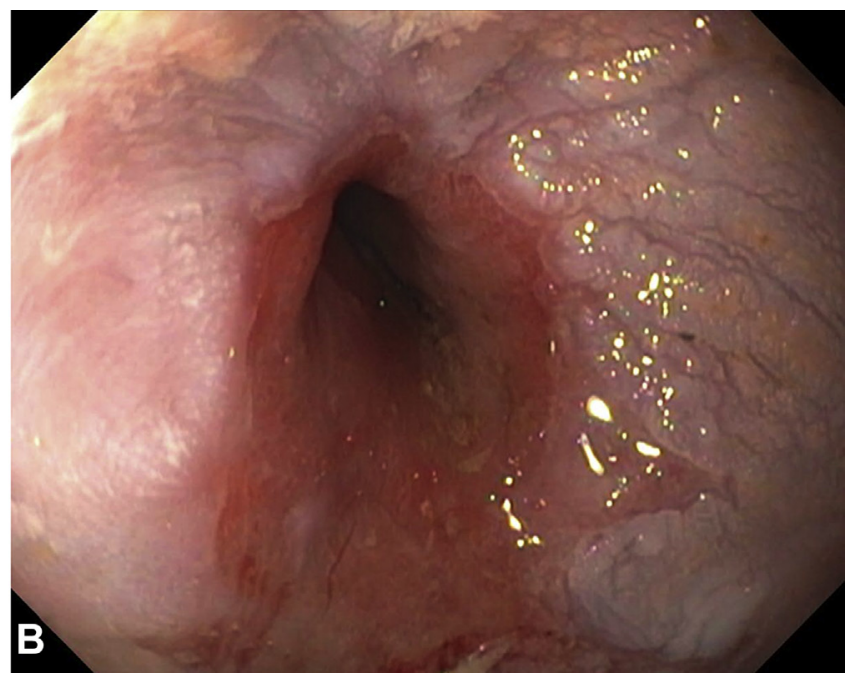

Figure 1. A, Marked dilation and retained food material in megaesophagus. B, Nonhypertonic and lax gastroesophageal junction.

Written transcript of the video audio is available online at www.VideoGIE.org. 


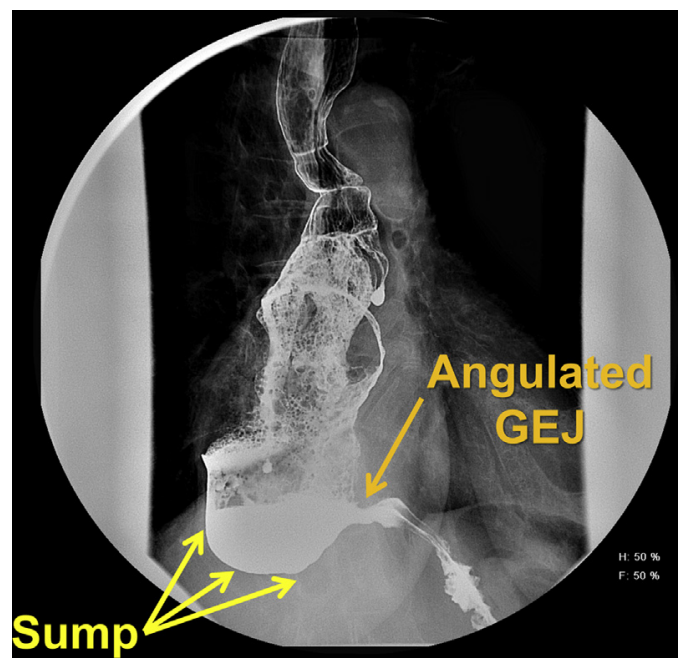

Figure 2. Sigmoid esophagus with sump formation and preferential contrast material pooling proximal to angulated gastroesophageal junction (GEJ).

1 day and 1 month, respectively, after the procedure (Video 1, available online at www.VideoGIE.org).

Esophagrams 24 hours and 1 month, respectively, after the procedure showed sustained reduction of the sump and improved esophageal emptying through the GEJ (Fig. 3). At a 6-month follow-up visit, the patient remained clinically well, tolerating at least a soft diet without dysphagia or regurgitation.

In conclusion, endoscopic esophagoplasty is a promising novel technique for end-stage achalasia megaesophagus with sump formation in patients deemed to be poor surgical candidates for esophagectomy. Long-term follow-up will determine the durability and sustained efficacy of the procedure.

\section{DISCLOSURE}

All authors disclosed no financial relationships relevant to this publication.

Abbreviation: GEJ, gastroesophageal junction.

\section{REFERENCES}

1. Orringer MB, Stirling MC. Esophageal resection for achalasia: indications and results. Ann Thoracic Surg 1989;47:340-5.

2. Bazerbachi F, Ravi K, Blackmon SH, et al. Endoscopic esophagoplasty for megaesophagus with sump stasis in end-stage achalasia [Abstract]. Gastrointest Endosc 2017;85:AB112.

Division of Gastroenterology and Hepatology (1); Division of Thoracic Surgery (2), Mayo Clinic, Rochester, Minnesota.

Copyright (c) 2017 American Society for Gastrointestinal Endoscopy. Published by Elsevier Inc. This is an open access article under the CC BYNC-ND license (http://creativecommons.org/licenses/by-nc-nd/4.0/).
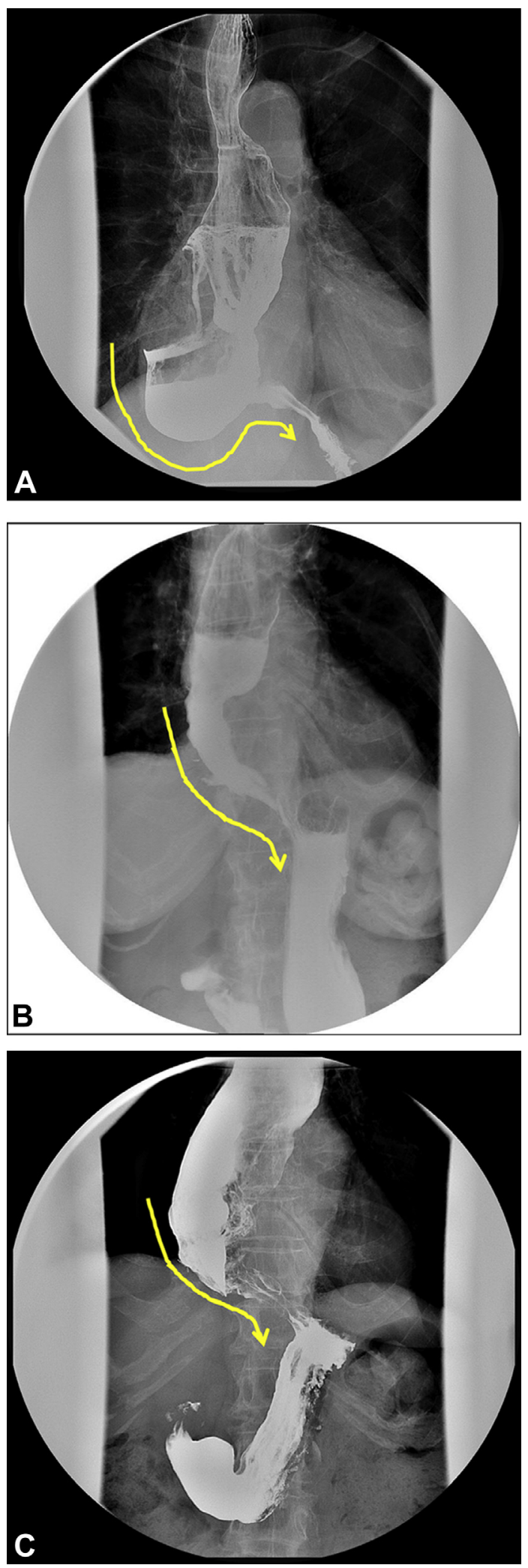

Figure 3. Esophagrams. A, Before esophagoplasty. B, 1 day after esophagoplasty. C, 1 month after esophagoplasty. 